\title{
Políticas institucionales de prevención de los problemas de salud generados por el consumo de bebidas alcohólicas en España y Europa
}

\author{
RoBledo, T. \\ Dirección General de Salud Pública y Consumo. Ministerio de Sanidad y Consumo.
}

Enviar correspondencia: Teresa Robledo de Dios. Jefe del Servicio de Promoción de Hábitos Saludables. Subdirección General de Promoción de la Salud y Epidemiología. Dirección General de Salud Pública y Consumo. Ministerio de Sanidad y Consumo. Paseo del Prado, 18-20. 28071 Madrid. Tel. 91 5964168/67. Fax 91 5964195. E-mail: trobledo@msc.es

\section{RESUMEN}

Los problemas relacionados con el consumo de alcohol y las motivaciones que inducen a beber son múltiples y complejas como lo es también su abordaje, que debe sustentarse en una política global, multisectorial y multidisciplinar, con la participación de todas las áreas implicadas: Salud, Educación, Bienestar Social, Empleo, Tráfico, Agricultura, Comercio, Justicia y Hacienda.

En España, la descentralización producida en materia de Sanidad e Higiene (art. 148.21 de la Constitución española), conlleva la necesidad de cooperación y coordinación entre las distintas Administraciones Sanitarias. Con dicha finalidad la Ley General de Sanidad previó la creación del Consejo Interterritorial del Sistema Nacional de Salud. Probablemente, la finalización del proceso de trasferencias sanitarias facilitará todo el proceso de coordinación y cooperación, que cada vez se manifiesta como más necesario.

En el Sistema Nacional de Salud, la intervención en el campo del alcohol es prioritaria, y así viene recogida en los distintos planes y políticas de salud que a nivel nacional, autonómico y local se están llevando a cabo. Las 17 Comunidades Autónomas han aprobado sus respectivos planes de salud y en todos ellos el alcohol es un área específica de intervención. Las actuaciones y programas sobre alcohol se llevan a cabo en algunas Comunidades desde los Planes Regionales de Drogodependencias y en otras desde el área de Salud Pública.

Se recogen los principales objetivos de las distintas actuaciones preventivas contempladas en los distintos planes y políticas de salud, y se presentan las actuaciones que, en el área de prevención de los problemas relacionados con el alcohol, viene desarrollando el Ministerio de Sanidad y Consumo, la Oficina Regional Europea de la Organización Mundial de la Salud y la Unión Europea.

Palabras clave: alcohol, prevención, salud pública, política sanitaria.

\begin{abstract}
The problems associated with alcohol consumption and the reasons to drink alcohol are multiple and complex, just like the ways to solve them, which must be based on comprehensive, multi-sectorial and multidisciplinary policies, with the collaboration of all the areas involved: Health, Education, Social Welfare, Employment, Traffic, Agriculture, Commerce, Justice and Finance.

In Spain, the decentralisation of the Health and Hygiene powers (Art. 148.21 of the Spanish Constitution) requires the continuous co-operation and co-ordination among the Health Authorities of the Autonomous Communities. Consequently, the General Health Law established the Inter-territorial Council of the National Health System. Probably, the completion of the above mentioned decentralisation process will favour the essential co-ordination and co-operation process.

Alcohol prevention is a priority within the National Health System, as it is demonstrated by the numerous plans and policies carried out at the national, autonomous and local level. The 17 Autonomous Communities have approved their respective health plans, and all of them include alcohol as a specific intervention area. Some Communities implement alcohol actions and programmes as part of their Regional Drug-dependencies Plans and other Public Health programmes.

This chapter explains the main objectives of the various prevention actions included in the different health plans and policies. Besides, we present the actions related with the prevention of alcohol-related problems implemented by the Ministry of Health and Consumer Affairs, the WHO European Region and the European Union.
\end{abstract}

Key words: alcohol, prevention, public health, health policies. 


\section{INTRODUCCIÓN}

C omo ya ha quedado de manifiesto en capítulos anteriores estamos hablando de un problema de importantes proporciones para la Salud Pública, no sólo de España sino de toda la Región Europea en su conjunto.

España es uno de los países donde el consumo de bebidas alcohólicas y los problemas relacionados con el mismo adquieren una gran importancia, no sólo por el alto nivel de producción de algunos tipos de bebidas alcohólicas $\left(3^{\circ}\right.$ país en producción mundial de vino con 36.080 millones de hectolitros y el $10^{\circ}$ en producción mundial de cerveza con 24.773 millones de hectolitros), sino también por la elevada prevalencia de su consumo (séptimo lugar en el consumo mundial de alcohol, con 10,1 litros de alcohol puro per cápita) ${ }^{1}$ y el amplio arraigo social que este hábito tiene en nuestra comunidad. Podemos hablar de una percepción casi generalizada de que el alcohol forma parte del patrón alimentario y dietético de nuestra sociedad.

Debemos destacar que, igual que ha ocurrido en otros países del Sur de Europa, el consumo de bebidas alcohólicas ha disminuido de manera notable en los últimos años.

Según las últimas Encuestas Nacionales de Salud ${ }^{2,3}$ más del $60 \%$ de la población española de 16 y más años, declara consumir habitualmente alguna cantidad de alcohol, mientras que aproximadamente un $4 \%$ son bebedores de riesgo.

El alcohol es la sustancia psicoactiva más consumida entre los escolares españoles. Según la última Encuesta sobre Drogas realizada a escolares entre 14 y 18 años (2000) el $76 \%$ declara haber consumido alcohol alguna vez y un $58 \%$ declara un consumo habitual (últimos 30 días). Los episodios de consumo abusivo de alcohol son relativamente frecuentes en los escolares, así el 39,7\% se ha emborrachado alguna vez, pese a lo cuál tan sólo un $7,4 \%$ de los escolares percibe que bebe mucho o bastante. La edad media de inicio al consumo de alcohol se sitúa en los 13,6 años ${ }^{4}$.

Debemos tener presente que pese a que probablemente sea la droga más consumida, no es un hábito generalizado. El porcentaje de jóvenes abstemios (no bebedores) se viene incrementando en los últimos años, al menos en España. Tal vez, el problema radica en el hecho de que los jóvenes que beben lo hacen en cantidades cada vez más elevadas, siendo cada vez más frecuente consumos muy elevados de alcohol en cortos períodos de tiempo.

Es evidente que hay una tendencia Europea a la homogenización de los patrones juveniles de consumo de alcohol. La globalización de los medios de comunicación y de los mercados está determinando, cada vez más, las percepciones de la gente, sus elecciones y comportamientos. Hoy en día muchos jóvenes tienen mayores oportunidades y disponen de más ingresos, pero son más vulnerables a las técnicas de marketing y ventas, cada vez más agresivas en los productos de consumo y sustancias potencialmente peligrosas como el alcohol. De ahí la importancia de incidir en estos factores que determinan el inicio al consumo ${ }^{5}$.

\section{POLÍTICAS INSTITUCIONALES}

Desde finales de los 60, los problemas derivados del consumo de alcohol se enfocaron desde la perspectiva de la Salud Pública, concentrando la atención en los individuos que sufrían alcoholismo y problemas relacionados con el alcohol, en el consumo general de alcohol en comunidades bebedoras, en los factores que inciden en ese consumo, en la relación entre distintos grados de consumo de alcohol y desarrollo de enfermedades relacionadas con el mismo y en los accidentes entre las personas bebedoras en su conjunto ${ }^{6}$.

De hecho, hoy en día podemos afirmar, como numerosos estudios comparativos internacionales así lo han puesto de manifiesto, que existe una relación dosis-respuesta 
entre el consumo de alcohol, y la frecuencia y gravedad de numerosas enfermedades. $Y$ que a mayores niveles de consumo corresponden tasas de mortalidad y morbilidad más elevadas de cirrosis hepática, determinados cánceres, accidentes, síndrome alcohólico fetal y otras enfermedades ${ }^{7.8}$.

Es este conocimiento científico de los factores y/o riesgos asociados al consumo de alcohol, lo que ha contribuido a fomentar el debate público sobre sus consecuencias. En el momento actual, todos los representantes nacionales del Plan Europeo de Actuación sobre Alcohol, de la Oficina Regional para Europa de la Organización Mundial de la Salud (OMS), coincidimos con Edwards en el hecho de que para conseguir una reducción significativa de los problemas relacionados con el alcohol es necesario un enfoque poblacional, dirigido a la reducción del consumo global (consumo per cápita), y un enfoque de alto riesgo dirigido a los bebedores excesivos, siendo siempre ambas estrategias complementarias ${ }^{9}$.

La necesidad de un enfoque poblacional viene justificada por el hecho, también constatado en España ${ }^{10}$, de que un cambio en el consumo total de alcohol en la población va acompañado de un cambio en igual dirección en la proporción de grandes bebedores. Por otro lado, dado que el abuso de alcohol aumenta la probabilidad de problemas físicos, psicológicos y sociales, el consumo medio ha de estar estrechamente relacionado con la prevalencia de estos problemas en la sociedad.

Incluso, tal y como recoge Eurocare en un informe recientemente elaborado para la Unión Europea ", los problemas derivados del consumo de alcohol en la familia, no menos importantes, están afectados por los mismos factores que inciden en los problemas que señalábamos anteriormente. El informe citado señala que la probabilidad de sufrir problemas familiares se incrementa con la cantidad de alcohol consumida y con la frecuencia de intoxicaciones. Y por tanto, las políticas tendentes a incrementar el consumo de alcohol tienden probablemente a incrementar los problemas derivados del alcohol en la familia, provocando daño y ruptura de la unidad familiar.

En realidad los patrones de uso y abuso del alcohol han variado en los últimos años. Diversas transformaciones y cambios económicos, culturales y sociales han coincidido en momentos determinados, provocando, entre otras cosas, un aumento de la disponibilidad del alcohol, con variación no sólo de la cantidad, sino de la calidad de las bebidas y un aumento del consumo por parte de la población femenina, juvenil e incluso adolescente.

En definitiva, los problemas relacionados con el consumo de alcohol y las motivaciones que inducen a beber son múltiples y complejas como lo es también su abordaje, que debe sustentarse en una política global, multisectorial y multidisciplinar, con la participación de todas las áreas implicadas: Salud, Educación, Bienestar Social, Empleo, Tráfico, Agricultura, Comercio, Justicia y Hacienda ${ }^{12}$.

\section{ESPAÑA: POLITICAS INSTITUCIONALES}

De acuerdo con la Constitución española y en materia sanitaria, el Estado tiene competencia exclusiva en "Sanidad exterior. Bases y coordinación general de la sanidad. Legislación sobre productos farmacéuticos. Legislación básica y régimen económico de la Seguridad Social" (artículo 149.1.16ª/17ª).

El establecimiento en la Constitución Española del Estado de las Autonomías, las cuales asumen a través de sus estatutos determinadas competencias que antes eran del Estado, obliga a incorporar en la Administración mecanismos de Coordinación, que la propia Constitución recoge. La descentralización producida en materia de Sanidad e Higiene (art. 148.21 de la Constitución española), ha conllevado en todo momento la necesidad de cooperación y coordinación entre las distintas Administraciones Sanitarias.

A partir del referente constitucional, la Ley General de Sanidad diseñó un modelo organizativo a través de un Sistema Nacional de 
Salud, concebido como el conjunto de Servicios de Salud bajo la responsabilidad principal de las Comunidades Autónomas, coordinados entre sí. En virtud de la competencia de "Coordinación General de la Sanidad" que posee el Estado de manera específica, éste creó, por la Ley General de Sanidad, el Consejo Interterritorial del Sistema Nacional de Salud, para asignarle los cometidos propios de la función coordinadora.

Según el artículo 70.2 de la Ley General de Sanidad, la Coordinación General Sanitaria incluye:

a) El establecimiento con carácter general de índices o criterios mínimos básicos y comunes para evaluar las necesidades de personal, centros o servicios sanitarios, el inventario definitivo de recursos institucionales y de personal sanitario y los mapas sanitarios nacionales.

b) La determinación de fines u objetivos mínimos comunes en materia de prevención, protección, promoción y asistencia sanitaria.

c) El marco de actuaciones y prioridades para alcanzar un sistema coherente, armónico y solidario y

d) El establecimiento con carácter general de criterios mínimos básicos y comunes de evaluación de la eficacia y rendimiento de los programas, centros o servicios sanitarios.

Un análisis crítico y objetivo de los mecanismos de cooperación y coordinación que han existido, así como de la realidad actual, nos lleva a la necesidad de una actualización, o probablemente a un replanteamiento global de dicho aspecto.

Probablemente la reflexión principal que podemos hacer es que hoy día, quince años después de la promulgación de la Ley General de Sanidad, sigue pendiente el proceso de coordinación por parte del Estado. Podemos hablar de una percepción casi generalizada de que es necesario un fortalecimiento del Consejo Interterritorial a través de mecanismos legales y de apoyos técnicos estructurados.
La Salud Pública puede ser un ejemplo de lo que significa la labor de coordinación del Consejo Interterritorial, calificándose de positiva la adopción de políticas de salud comunes a problemas comunes, sobre todo cuando las soluciones son a largo o medio plazo.

La creación, en 1992, de la Comisión de Salud Pública ha supuesto un avance en la medida que ha posibilitado la creación de una Red Nacional de Vigilancia Epidemiológica, la aplicación de directivas comunitarias en materia alimentaria, se ha trabajado hacia una unificación de los calendarios vacunales, se ha creado un Grupo de Promoción de la Salud..., aunque también se le puede achacar una insuficiente agilidad y falta de mecanismos de decisión suficientemente ejecutivos en los casos de emergencias.

Probablemente, la finalización del proceso de trasferencias sanitarias, así como la articulación de mecanismos de financiación sólidos, duraderos y transparentes facilitará todo este proceso de coordinación y cooperación.

Centrándonos en el tema que nos ocupa podemos decir que, en España, la intervención en el campo del alcohol es prioritaria, y así viene recogida en los distintos planes y políticas de salud que a nivel nacional, autonómico y local se están llevando a cabo.

Las 17 Comunidades Autónomas han aprobado sus respectivos planes de salud (Tabla 1). En todos ellos el alcohol es un área específica de intervención, si bien, la formulación de los objetivos varía mucho de unos planes a otros, pudiendo algunos planes contemplar este tema en un solo objetivo general, mientras que en otros se presenta con varios objetivos generales e incluso detallados objetivos específicos.

En lo referente a la dependencia orgánica las actuaciones y programas sobre alcohol se llevan a cabo en algunas Comunidades desde los Planes Regionales de Drogodependencias y en otras desde el área de Salud Pública.

Las principales actuaciones preventivas contempladas hacen referencia a los siguientes objetivos: 


\section{Tabla 1. Los planes de salud en las CC.AA. y en el Ministerio de Sanidad y Consumo}

\begin{tabular}{|c|c|c|c|c|}
\hline COMUNIDAD & PLAN & $\begin{array}{l}\text { CONSEJO } \\
\text { INTERTERRITORIAL }\end{array}$ & AÑO & OBSERVACIONES \\
\hline Andalucía & si & 27.9 .93 & 1993 & Aprobado por Consejo de Gobierno, enviado al Parlamento \\
\hline Aragón & Reelabor. & & 1992 & Existe un borrador enviado para sugerencias \\
\hline Asturias & $\mathrm{Si}$ & & 1997 & Estrategias de Salud \\
\hline Baleares & $\mathrm{Si}$ & & Sin fecha & Documento previo. Pendiente de aprobación por Parlamento \\
\hline Canarias & $\mathrm{Si}$ & & Sin fecha & Plan de Salud 1996-2000 \\
\hline Cantabria & $\mathrm{Si}$ & & 1996 & $\begin{array}{l}\text { Aprobado por Consejo de Gobierno en 12.96. Plan de Salud } \\
1996-2000\end{array}$ \\
\hline Castilla-La Mancha & Si & & 1995 & $\begin{array}{l}\text { Aprobado por Consejo de Gobierno en 4.95. Plan de Salud } \\
\text { 1995-2000 }\end{array}$ \\
\hline Castilla y León & $\mathrm{Si}$ & & 1998 & Aprobado por Consejo de Gobierno el 8 de octubre de 1998 \\
\hline Cataluña & $\mathrm{Si}$ & & 1997 & $\begin{array}{l}\text { En continuidad y coherencia con el anterior Plan de Salud } \\
\text { 1996-1998 }\end{array}$ \\
\hline C. Valenciana & Revisión & & 1995 & Es un Documento considerado base \\
\hline Extremadura & $\mathrm{Si}$ & & 1997 & Aprobado en Consejo de Gobierno. Plan de Salud 1997-2000 \\
\hline Galicia & $\mathrm{Si}$ & & 1998 & Segundo Plan aprobado \\
\hline Madrid & $\mathrm{Si}$ & & 1995 & En revisión y actualización de objetivos \\
\hline Murcia & $\mathrm{Si}$ & & 1998 & Plan de Salud 1998-2000 \\
\hline Navarra & $\mathrm{Si}$ & 17.12 .90 & 1991 & \\
\hline País Vasco & $\mathrm{Si}$ & 20.12 .93 & 1994 & Aprobado por Consejo de Gobierno, remitido Parlamento \\
\hline Rioja & $\mathrm{Si}$ & & 1997 & \\
\hline $\mathrm{M}^{0}$. Sanidad y C. & $\mathrm{Si}$ & 6.11 .95 & 1995 & Informado por el Pleno del CISNS de 6.11 .95 \\
\hline
\end{tabular}

- Limitaciones a la venta y consumo de alcohol.

- Promover hábitos saludables de vida.

- Informar y educar a la población en general y a grupos poblacionales específicos (niños, adolescentes, embarazadas) acerca de los riesgos derivados del consumo de alcohol.

- Promover la participación intersectorial.

- Educar para la Salud en medio escolar.

- Concienciar a la población acerca de la necesidad de un consumo moderado de alcohol y

- Desarrollar intervenciones especiales en grupos de riesgo.

Estos objetivos son asumidos por el Ministerio de Sanidad y Consumo, que en el área de prevención de los problemas relacionados con el alcohol, viene desarrollando las siguientes actuaciones:

\subsection{MEDIDAS DE REDUCCIÓN DE LA DISPONIBILIDAD Y ACCESIBILIDAD.}

Se contemplan un conjunto de medidas de promoción y de protección de la salud, (legales, económicas, ambientales, etc...), que tienden a construir ambientes favorables que faciliten comportamientos saludables. Hablamos de:

\section{- Limitaciones a la venta y consumo de alcohol.}

Las limitaciones de edad representan una barrera legal para acceder al alcohol. Hoy día podemos afirmar que cuánto más se retrase la edad legal de acceso al alcohol, más se retrasará la edad de inicio en el consumo de alcohol.

En la Unión Europea los límites legales de edad varían de los 15 a los 20 años, según el Estudio ECAS ${ }^{14}$ (que analiza todos los países de la UE con la exclusión de Luxemburgo e inclusión de Noruega) 
(Tabla 2). En España la edad legal límite para la venta y consumo de alcohol en lugares públicos es de 16 años a nivel nacional, habiéndose incrementado a 18 años en algunas Comunidades Autónomas (Tabla 3).

El Ministerio de Sanidad y Consumo tiene la voluntad de elevar a 18 años la edad legal límite para la venta de alcohol, unificando por tanto este límite en todo el territorio nacional.

\section{- Control de la publicidad.}

En materia de control de la publicidad tenemos prohibida, desde 1.988, la publicidad en televisión de las bebidas alcohólicas de más de $20^{\circ}$, así como traspuesto a nuestro ordenamiento jurídico la directiva comunitaria sobre radiodifusión televisiva.

Algunas Comunidades Autónomas han ampliado estas limitaciones a otros medios (radio y televisión en determinados tramos horarios, publicidad exterior, publicidad en el interior de determinados establecimientos, revistas juveniles, patrocinio de acontecimientos culturales y deportivos juveniles...).

\section{- Impuestos especiales}

La recaudación por impuestos especiales en concepto de alcohol, bebidas derivadas y cerveza supuso el $7 \%$ de todos los ingresos obtenidos por impuestos especiales de fabricación en 1998. Existe una tendencia creciente de los impuestos especiales que gravan el alcohol y bebidas derivadas ${ }^{15}$. En la tabla 4 se presenta la recaudación por este concepto en los últimos años.

\subsection{MEDIDAS DE INFORMACIÓNY EDU- CACIÓN PARA LA SALUD}

Educar para la Salud en medio escolar, promover hábitos saludables de vida, informar y educar a la población en general y a grupos poblacionales específicos (niños, adolescentes, embarazadas) acerca de los riesgos derivados del consumo de alcohol y concienciar a la población acerca de la necesidad de un consumo moderado de alcohol, son objetivos de todas las Administraciones Sanitarias.

- En este sentido y desde 1.989, se viene trabajando, a través de un convenio de

Tabla 2. Edad legal límite para la venta de alcohol en Europa, en el año 2000

\begin{tabular}{|c|c|c|c|c|c|c|}
\hline & \multicolumn{3}{|c|}{$\begin{array}{c}\text { Venta donde no se consume } \\
\text { (Off premisse) }\end{array}$} & \multicolumn{3}{|c|}{$\begin{array}{l}\text { Venta donde se consume } \\
\text { (On premisse) }\end{array}$} \\
\hline & Cerveza & Vino & Destiladas & Cerveza & Vino & Destiladas \\
\hline Austria & 16 & 16 & 18 & 16 & 16 & 18 \\
\hline Bélgica & 16 & 16 & 18 & 16 & 16 & 18 \\
\hline Dinamarca & 15 & 15 & 15 & 18 & 18 & 18 \\
\hline Finlandia & 18 & 18 & 20 & 18 & 18 & 18 \\
\hline Francia & 16 & 16 & 16 & 16 & 16 & 16 \\
\hline Alemania & 16 & 16 & 18 & 16 & 16 & 18 \\
\hline Grecia & - & - & 18 & - & - & 18 \\
\hline Irlanda & 18 & 18 & 18 & 18 & 18 & 18 \\
\hline Italia & 16 & 16 & 16 & 16 & 16 & 16 \\
\hline Holanda & 16 & 16 & 18 & 16 & 16 & 18 \\
\hline Noruega & 18 & 18 & 20 & 18 & 18 & 20 \\
\hline Portugal & - & - & - & 16 & 16 & 16 \\
\hline España & 16 & 16 & 16 & 16 & 16 & 16 \\
\hline Suecia & 18 & 20 & 20 & 18 & 18 & 18 \\
\hline Inglaterra & 18 & 18 & 18 & 16 & 16 & 18 \\
\hline
\end{tabular}

Fuente: ECAS Study. Febrero 2001. 


\section{Tabla 3. Edad legal límite para la venta de bebidas alcohólicas en ámbito nacional y autonómico. Año 2001}

\begin{tabular}{|c|c|c|}
\hline & EDAD LEGAL LÍMITE & NORMA QUE LO REGULA \\
\hline España & 16 años & $\begin{array}{l}\text { Real Decreto 2816/1992, de } 27 \text { de agosto, Reglamento } \\
\text { General de Espectáculos y actividades Recreativas. }\end{array}$ \\
\hline Andalucía & 18 años & $\begin{array}{l}\text { Ley 4/1997, de } 9 \text { de julio, de Prevención y Asistencia en } \\
\text { materia de Drogas. }\end{array}$ \\
\hline Aragón & 18 años & $\begin{array}{l}\text { Ley } 3 / 2001 \text {, de } 4 \text { abril, de prevención, asistencia y rein- } \\
\text { serción social en materia de drogodependencias. }\end{array}$ \\
\hline Asturias & 16 años & $\begin{array}{l}\text { Ley 5/1990, de } 19 \text { diciembre, sobre prohibición de } \\
\text { venta de bebidas alcohólicas a menores de } 16 \text { años. }\end{array}$ \\
\hline Canarias & 18 años & $\begin{array}{l}\text { Ley } 1 / 1997 \text {, de } 7 \text { de febrero, de Atención Integral a los } \\
\text { menores. }\end{array}$ \\
\hline Cantabria & 18 años & $\begin{array}{l}\text { Ley de Cantabria 5/1997, de } 6 \text { octubre, de Prevención, } \\
\text { Asistencia e Incorporación Social en Materia de Drogo- } \\
\text { dependencias. }\end{array}$ \\
\hline Castilla León & $\begin{array}{l}16 \text { años. }{ }^{*} 18 \text { en el caso de bebidas } \\
\text { alcohólicas de más de } 18^{\circ} \text { centesimales) }\end{array}$ & $\begin{array}{l}\text { Ley 3/1994, de } 29 \text { de marzo, de Prevención, Asistencia } \\
\text { e Integración Social de Drogodependientes de Castilla } \\
\text { y León. }\end{array}$ \\
\hline Castilla La Mancha & 18 años & $\begin{array}{l}\text { Ley 2/1995, de } 2 \text { de marzo, contra la Venta y Publicidad } \\
\text { de Bebidas Alcohólicas a Menores. }\end{array}$ \\
\hline Cataluña & $\begin{array}{l}16 \text { años. (*18 en el caso de bebidas } \\
\left.\text { alcohólicas de más de } 23^{\circ}\right)\left.\right|^{*} 18 \text { años } \\
\text { en el horario de } 24 \text { horas a } 6 \text { horas) }\end{array}$ & $\begin{array}{l}\text { Ley } 10 / 1991 \text {, de } 10 \text { de mayo, de modificación de la Ley } \\
\text { 20/1985, de prevención y asistencia en materia de sus- } \\
\text { tancias que pueden generar dependencia. }\end{array}$ \\
\hline $\begin{array}{l}\text { Comunidad } \\
\text { Valenciana }\end{array}$ & $\begin{array}{l}16 \text { años. (*18 en el caso de bebidas } \\
\left.\text { alcohólicas de más de } 18^{\circ}\right)\end{array}$ & $\begin{array}{l}\text { Ley } 3 / 1997 \text {, de } 16 \text { de junio, sobre drogodependencias y } \\
\text { otros trastornos adictivos. }\end{array}$ \\
\hline Extremadura & 18 años & $\begin{array}{l}\text { Ley 4/1997, de } 10 \text { de abril, de Medidas de Prevención y } \\
\text { Control de la Venta y Publicidad de Bebidas Alcohólicas } \\
\text { para Menores de Edad. }\end{array}$ \\
\hline Galicia & $\begin{array}{l}16 \text { años. }{ }^{*} 18 \text { en el caso de bebidas } \\
\text { alcohólicas de más de } 18^{\circ} \text { centesimales) }\end{array}$ & Ley 2/1996, de 8 de mayo, de Galicia, sobre drogas. \\
\hline Madrid & 18 Años & $\begin{array}{l}\text { Ley } 5 / 2000 \text {, de } 8 \text { de mayo, por la que se eleva la edad } \\
\text { mínima de acceso a las bebidas alcohólicas }\end{array}$ \\
\hline Murcia & 18 Años & $\begin{array}{l}\text { Ley } 6 / 1997, \text { de } 2 \text { de octubre, sobre drogas para la pre- } \\
\text { vención, asistencia e integración social. }\end{array}$ \\
\hline Navarra & 18 Años & $\begin{array}{l}\text { Ley Foral 10/1991, de } 16 \text { de marzo, sobre prevención y } \\
\text { limitación de consumo de bebidas alcohólicas por } \\
\text { menores de edad. }\end{array}$ \\
\hline País Vaso & 18 Años & $\begin{array}{l}\text { Ley 18/1998, de } 25 \text { de junio, sobre Prevención, Asis- } \\
\text { tencia e Inserción en materia de Drogodependencias. }\end{array}$ \\
\hline La Rioja & 18 Años & $\begin{array}{l}\text { Ley } 4 / 2000 \text {, de } 25 \text { de octubre, de Espectáculos Públi- } \\
\text { cos y Actividades Recreativas de la Comunidad Autó- } \\
\text { noma de la Rioja. }\end{array}$ \\
\hline
\end{tabular}

Elaboración: Subdirección General de Promoción de la Salud y Epidemiología. Dirección General de Salud Pública y Consumo. Ministerio de Sanidad y Consumo 
Tabla 4. Evolución de la recaudación por impuestos especiales

\begin{tabular}{lrrrr}
\hline & $\mathbf{1 9 9 5}$ & $\mathbf{1 9 9 6}$ & $\mathbf{1 9 9 7}$ & \multicolumn{1}{c}{$\mathbf{1 9 9 8}$} \\
\hline Alcohol y Bebidas Derivadas & 87.238 & 99.482 & 93.015 & 114.533 \\
Productos Intermedios & 3.826 & 4.412 & 3.916 & 3.605 \\
Cerveza & 31.664 & 33.115 & 27.218 & 29.574 \\
\hline
\end{tabular}

Millones de pesetas.

Fuente: Estudio sobre los impuestos especiales en 1988. Departamento de Aduanas e impuestos especiales.

colaboración entre el Departamento de Sanidad y el de Educación y Ciencia e Interior, en las áreas de Educación para la Salud, Formación y Desarrollo Profesional e Investigación. Podemos señalar la realización de campañas y concursos escolares de carteles, cómics y videos, la elaboración de materiales didácticos, tanto para el profesor como para el alumno, así como la elaboración de guías y folletos y la puesta en marcha de programas de entrenamiento en habilidades de vida (Construyendo Salud).

En noviembre de 1996 los Ministerios de Educación y Cultura, Sanidad y Consumo e Interior (Plan Nacional sobre Drogas), firmaron un protocolo de intenciones de colaboración para promover la Educación para la Salud en la escuela, con el objetivo de promover actuaciones que contribuyeran al desarrollo de estilos y hábitos de vida saludables.

El Programa "Construyendo Salud" es el resultado de la ampliación y adaptación en nuestro país del Programa "Entrenamiento en Habilidades de Vida" desarrollado por el Doctor Gilbert J. Botvin de la Universidad de Cornell (Nueva York); dicha adaptación ha sido realizada por un equipo de profesores de la Universidad de Santiago de Compostela. El Programa "Construyendo Salud" constituye una aproximación a la prevención, que se centra en los principales factores sociales y psicológicos que promueven las primeras etapas del uso y abuso de drogas y las actividades antisociales. Por eso, presta una gran atención al desarrollo de habilidades personales y a la aplicación de estas actividades a situaciones concretas en las que se pueden experimentar presiones hacia el consumo de drogas o a la implicación en actividades antisociales.

El programa consiste en el desarrollo en el aula de una serie de contenidos relacionados con dichos temas, de una forma participativa, mediante dinámicas de grupo, por profesorado que ha sido específicamente entrenado para esta tarea. Se han editado un libro del profesor, otro del alumno y un diario de implantación para cada uno de los dos años durante los que se desarrolla el programa.

Paralelamente a este programa, se desarrolla otro "Programa de Puertas Abiertas", centrado en actividades de ocio y tiempo libre, dirigidas al alumno en horario no lectivo, Ilevado a cabo por ONG especializadas: Asociación Deporte y Vida, Carpe Diem y Cruz Roja Juventud. Todas estas actividades tiene como objetivo favorecer una utilización sana y positiva del tiempo libre incompatible con el uso de drogas.

Este programa se está aplicando en más de 120 centros seleccionados en zonas con déficit socioeconómico y cultural y en él han participado 9.000 escolares y 700 profesores (datos de octubre de 1999).

La evaluación de este programa, en 1999, fue muy positiva. En cuanto a los resultados del programa hay que destacar en relación al alcohol:

- La intervención logra reducir en un 33\% el número de alumnos que comienzan a consumirlo. 
- Los alumnos que ya se habían iniciado disminuyen de forma significativa dicho consumo.

Las transferencias educativas a las Comunidades Autónomas implican la necesidad de rediseñar la organización de la aplicación del "Programa Construyendo Salud". El objetivo actual de los tres Ministerios es seguir aplicándolo, añadiendo un nuevo elemento, además del componente escolar y extraescolar, que es el componente familiar del programa, a través del ofrecimiento de escuelas de padres y madres. También está previsto desarrollar una asesoría técnica a través de Internet, para que el desarrollo de este programa pueda extenderse y optimizarse en cualquier centro educativo que quiera desarrollarlo. Por último, queda decir que este programa ha sido el estímulo para que se inicie la evaluación de programas en esta materia, evaluaciones que nos permitirán seleccionar los programas más eficaces.

- También, venimos realizando diversas actividades dentro del área de salud y prevención del Plan de la Juventud, que tienen como objetivo principal la promoción entre los jóvenes de hábitos de vida saludables y la prevención de riesgos a los que están expuestos. Las actividades están dirigidas a influir en la formación de aptitudes y comportamientos de los jóvenes, fomentando sanos estilos de vida, creando corrientes de opinión que hagan frente a aquellas situaciones que mayores efectos negativos tienen entre los jóvenes: abuso de alcohol, tabaquismo, toxicomanías, accidentes de tráfico y embarazos no deseados. En este contexto debemos señalar la reciente edición de una Guía Informativa sobre Consumo Juvenil de Alcohol titulada ¿Porqué es importante hablar de Alcohol?.

- Además, en este año el Ministerio de Sanidad y Consumo, a través de la Dirección General de Salud Pública firmará un CONVENIO MARCO DE COLABORACIÓN CON EL INSTITUTO NACIONAL DE LA JUVENTUD (INJUVE), que intentará hacer frente a las situaciones anteriormente descritas.

- Finalmente, debemos destacar la gran importancia de las actuaciones realizadas por las Consejerías de Sanidad de las Comunidades Autónomas y por las Concejalías de Sanidad de numerosos municipios españoles, que están volcando grandes esfuerzos en la prevención de los problemas derivados del consumo juvenil de alcohol, como por ejemplo, a través del fomento de la promoción de la salud en el medio escolar y del desarrollo de actividades de ocio saludables, durante la tarde/noche de los fines de semana, organizadas y desarrolladas por y para los jóvenes en los polideportivos municipales, así como la organización de redes de transporte alternativo durante el fin de semana, en las "rutas" de diversión de los jóvenes.

\subsection{REDUCCIÓN DE DAÑOS}

- PREVENCIÓN DE LOS ACCIDENTES DE TRÁFICO RELACIONADOS CON EL CONSUMO DE ALCOHOL. Como iniciativas debemos destacar la participación del Departamento de Sanidad en:

- Comisión Interministerial de Seguridad Vial

- Consejo Superior de Tráfico y Seguridad de la Circulación Vial

- Participación en los Plenos y en el Grupo de trabajo GT14 encargado de la elaboración de los Planes Nacionales de Seguridad Vial.

- La reducción realizada de la tasa legal de alcoholemia, que actualmente se sitúa en 0,5 gramos/litro para turismos y 0,3 gr/litro para transporte de mercancías, vehículos de emergencia y conductores noveles durante los dos primeros años de carnet.

- Desarrollo de un Proyecto de Prevención de Accidentes de Tráfico en el ámbito sanitario de la Atención Primaria de Salud en colaboración con el Programa de Acti- 
vidades Preventivas y de Promoción de la Salud de la Semfyc, y la puesta en marcha de un estudio que valore el impacto de la prescripción de medicamentos en los Accidentes de Tráfico en el ámbito de la Atención Primaria de Salud.

- Dentro del ÁREA ASISTENCIAL es de destacar las importantes iniciativas realizadas en España, y dirigidas a la detección sistemática de bebedores de riesgo en el nivel de Atención Primaria de Salud, así como la promoción de las intervenciones breves (consejo sanitario) en este campo. Importantes iniciativas que son promovidas desde el INSALUD y desde Sociedades Científicas, como ocurre con el Programa de Actividades de Prevención y Promoción de la Salud de la Sociedad Española de Medicina de Familia y Comunitaria, programa al que el Ministerio apoya técnica y económicamente.

\subsection{COORDINACIÓN}

Además de la participación en Reuniones de Coordinación con Instituciones y Entidades Nacionales, como la Dirección General de Tráfico y el Plan Nacional sobre Drogas, y dado nuestro ámbito competencial señalado anteriormente, es una prioridad para nosotros potenciar las actuaciones de coordinación en el seno del Consejo Interterritorial.

Actualmente, estamos trabajando en un grupo de trabajo sobre jóvenes con las Comunidades Autónomas, creado en el seno del Grupo de Promoción de la Salud de la Comisión de Salud Pública del Consejo Interterritorial del SNS, para la elaboración de RECOMENDACIONES en relación con los principales problemas que afectan a la juventud española, entre ellos el alcohol.

\section{EUROPA: POLÍTICAS INSTITUCIONALES}

\subsection{PLAN EUROPEO DE ACTUACIÓN SOBRE ALCOHOL}

Además de la política de coordinación que comentábamos, un papel clave a desempeñar por la Administración del Estado es la representación de España en las políticas de salud desarrolladas por la Oficina Regional Europea de la Organización Mundial de la Salud (OMS) y de la Unión Europea.

Como miembros que somos de la Región Europea nos sentimos comprometidos con los Planes Europeos de Actuación sobre Alcohol, de OMS-Europa: Planes que sin duda, y desde 1992, se han convertido en un claro elemento dinamizador de políticas preventivas en los distintos Estados Miembros.

El planteamiento principal de estos planes se basa en el hecho de que los problemas relacionados con el consumo de alcohol y las motivaciones que incitan a beber son múltiples y complejas como lo es también su abordaje, que debe sustentarse en una política global, multisectorial y multidisciplinar. Por ello, no existe una "actuación estrella" para su abordaje, sino que toda estrategia de intervención necesita estar enmarcada en una política global, tal como se señala en la "Carta Europea sobre Alcohol", adoptada en Diciembre de 1995, por los Estados Miembros de la Región Europea de la Organización Mundial de la Salud en el marco del primer Plan Europeo de Actuación sobre Alcohol $(\mathrm{PEAA})^{16}$, en la que se recogen los principios éticos, metas, y diez estrategias para la acción sobre el alcohol. Carta Europea que sin duda constituye un hecho histórico sin precedentes, y que marca un punto de inflexión en el desarrollo de políticas de salud relacionadas con el alcohol en el ámbito de la Región Europea.

Las 10 estrategias para la acción, aprobadas con esta carta, sirven de nexo común para el desarrollo de actividades en el campo del alcohol, sin interferir con las propuestas que en los distintos ámbitos se desarrollen en función de las necesidades y prioridades.

EI primer PLAN EUROPEO DE ACTUACIÓN SOBRE ALCOHOL fue adoptado en 1992 por el Comité Regional para Europa de la OMS. Este plan fue concebido como un conjunto positivo de directrices para los Esta- 
dos Miembros, con el objetivo último de crear un movimiento europeo que respalde las actuaciones realizadas en los ámbitos local, nacional e internacional, y reduzca los riesgos para la salud y los problemas sociales que ocasiona el consumo de alcohol ${ }^{17}$.

Para garantizar un rápido impacto del Plan, la Oficina Regional para Europa de la OMS, propone el desarrollo de políticas saludables que consideren o reconsideren los aspectos legislativos sobre política en materia de alcohol, la intervención comunitaria y el desarrollo de iniciativas en proyectos actualmente en marcha (ciudades saludables, escuelas promotoras de salud) y el refuerzo de la actividad en los sistemas sanitarios y de asistencia social, especialmente en la atención primaria de salud.

En términos estratégicos este Plan se puso en marcha al mismo tiempo que el Segundo Plan de Actuación para una Europa libre de Tabaco y el programa de actividades en los países de Europa Central y Oriental para la prevención del consumo abusivo de drogas. De hecho, se creó una unidad combinada sobre Alcohol, drogas y Tabaco en la Oficina Regional para Europa de la OMS, con el objetivo de desarrollar estos puntos clave contenidos en la Carta de Ottawa.

Con objeto de fomentar la colaboración internacional, los Ministerios de Salud de los distintos Estados Miembros a instancias de la oficina regional de la OMS, designaron representantes nacionales para dicho Plan. Así mismo, fue promovida la traducción de dicho plan a 15 lenguas, incluido el castellano. Debemos destacar que en el transcurso de este primer PEAA han tenido lugar cinco encuentros de representantes nacionales (Viena, Berna, Roma, Madrid y Varsovia) y una Conferencia Europea Ministerial sobre Salud, Sociedad y Alcohol (París 1995).

En septiembre de 1.999 fue aprobado en Florencia, durante la $49^{\text {th }}$ reunión del Comité Regional de la OMS un segundo plan, el PLAN EUROPEO DE ACTUACIÓN SOBRE ALCOHOL 2000-2005 ${ }^{18}$, en cuyo contexto han tenido lugar dos encuentros de representantes nacionales y la Segunda Conferencia
Europea Ministerial sobre Alcohol, ALCOHOL Y JÓVENES (Estocolmo, febrero del 2001), conferencia en la que fue aprobada por todos los estados miembros de la Región Europea, una nueva declaración o carta europea sobre "Alcohol y Jóvenes" 19.

\section{Alcohol cuanto menos mejor}

En relación con el consumo de alcohol la OMS lanzó un mensaje: ALCOHOL- LESS IS

BETTER, traducido por nosotros como $A L$ COHOL: CUANTO MENOS MEJOR. Con este mensaje se pretende transmitir la idea de que no existe un límite estricto en relación al nivel de consumo de alcohol a partir del cual éste empieza a ser perjudicial para la salud, pero que en cualquier circunstancia cuanto menos cantidad de alcohol se consuma mejor.

Este mensaje es apoyado por el Ministerio de Sanidad y Consumo. No debemos olvidar que en una sociedad productora y consumidora como la nuestra hablar de los posibles efectos beneficiosos del alcohol, puede aumentar el riesgo de efectos adversos por un aumento del consumo entre los ya consumidores o por un incremento en el número de nuevos consumidores ${ }^{20}$.

\subsection{ESTRATEGIA COMUNITARIA}

Con fecha 24 de noviembre de 1993, la Comisión de las Comunidades Europeas emitió una Comunicación de vital importancia sobre el marco de actuación en el ámbito de la salud pública. En ella se definían ocho áreas prioritarias de intervención, que dieron lugar a ocho programas de intervención, entre los cuáles no se incluía el alcohol.

Podríamos decir que, salvo por una resolución del año 1986 sobre consumo abusivo de alcohol, no ha existido una política comunitaria en materia de salud pública y alcohol hasta hace unos años.

En junio de 1997 tuvo lugar en Luxemburgo la primera reunión de un grupo de trabajo sobre "Alcohol y Salud", fruto del trabajo de 
este grupo se elaboró una "Recomendación del Consejo sobre consumo de alcohol por parte de niños y adolescentes", aprobada por el Consejo de Ministros de Sanidad el pasado 5 de junio y que sin duda constituye un modesto pero importante avance en la construcción de una política comunitaria en materia de prevención de los problemas derivados del consumo de alcohol ${ }^{21}$.

La Recomendación del Consejo recoge la necesidad de que los distintos Estados Miembros, con el apoyo según proceda de la Comisión, deben formular estrategias y poner en marcha acciones reguladoras con respecto a los jóvenes y el alcohol, garantizando el desarrollo, aplicación y evaluación de políticas globales de promoción de la salud, dirigidas a este colectivo, así como de programas específicos. Asimismo, establece la necesidad de fomentar, en función de los distintos entornos jurídicos, normativos o autorreguladores, mecanismos eficaces de control en los sectores de la promoción, la comercialización y la distribución.

El 5 de junio del 2001 también fue aprobado un documento sobre "Conclusiones del Consejo sobre una Estrategia Comunitaria dirigida a reducir el daño derivado de los problemas de alcohol", en el que el Consejo invita a la Comisión a que desarrolle una estrategia comunitaria comprensiva dirigida a disminuir los problemas derivados del consumo de alcohol.

Sólo queda esperar que estas actuaciones se sigan desarrollando en el marco del futuro programa comunitario de Salud Pública, que casi con toda seguridad será en el 2002.

\section{BIBLIOGRAFIA}

(1) PRODUKTSCHAP VOOR GEDISTILLEERDE DRANKEN (1999) "World Drink Trends 1999". NTC Publications Ltd, Oxford.

(2) BIGLINO L, CORONADO A, GIL E, GIL J, PRIETO A, REGIDOR E (1994) "Encuesta Nacional de Salud 1993". Rev San Hig Pública 68:121-178.
(3) MINISTERIO DE SANIDADY CONSUMO (1998) "Encuesta Nacional de Salud de España 1997." Ministerio de Sanidad y Consumo, Madrid.

(4) PLAN NACIONAL SOBRE DROGAS (2001) "Encuesta sobre Drogas en la población escolar." Ministerio del Interior, Madrid.

(5) WORLD HEALTH ORGANIZATION (2001) "Declaration on Young People and Alcohol". WHO. Stockholm, 2001.

(6) GIL E, VARGAS F, ROBLEDO T, ESPIGA I (1994) "Alcohol y Salud Pública". Serie de Informes Técnicos $n^{\circ} 1$. Ministerio de Sanidad y Consumo, Madrid.

(7) LEIFMAN H, ROMELSJÖ A (1997) "The effect of changes in alcohol consumption on mortality and admissions with alcohol-related diagnoses in Stockholm County- a time series analysis" Addiction 92:1523-1536.

(8) SMART RG, MANN RE, SUURVALI H (1998) "Changes in Liver Cirrhosis Death Rates in Different Countries in Relation to Per Capita Alcohol Consumption and Alcoholics Anonymous Membership". J Stud Alcohol 59:245-249.

(9) EDWARDS G, ANDERSON P, BABOR TF, CASSWELL S, FERRENCE R, GIESBRETCH N, ET AL (1994) "Alcohol policy and the public good". Oxford University Press, Oxford.

(10) RODRIGUEZ-ARTALEJO F, DE ANDRÉS B, BANEGAS JR, GUALLAR-CASTILLÓN P, VILLAR F, DEL REY (1999) “La disminución del consumo moderado de alcohol se ha asociado a un descenso de los bebedores excesivos en España en el período 1987-1993". Med Clin 113:371-373.

(11) EUROCARE, COFACE (1998) "Alcohol Problems in the Family. A report to the European Union". World Health Organization Europe, England.

(12) GIL E, GONZALEZ J, VILLAR F (1999) "Informe sobre la salud de los Españoles: 1998." Ministerio de Sanidad y Consumo, Madrid.

(13) Ley 14/1986, de 25 de abril, General de Sanidad.

(14) Österberg E, Karlsson T (2001) "Trends in alcohol control policies in the EU Member States and Norway, 1950-2000". Conferencia de Estocolmo sobre Alcohol y Jóvenes, 2001.

(15) AGENCIA TRIBUTARIA. DEPARTAMENTO DE ADUANAS E IMPUESTOS ESPECIALES (1998) "Estudio sobre los impuestos especiales en 1998" Ministerio de Economía y Hacienda, Madrid. 
(16) GIL E, ROBLEDO T, RUBIO J, GARCIA M, VARGAS F (1995) "Plan Europeo de Actuación sobre Alcohol". Rev Esp Salud Pública 69:361-383.

(17) ROBLEDO T, ÁLVAREZ FJ (1996) “Plan Europeo de actuación sobre el consumo de alcohol: una mirada hacia el futuro". Med Clin 106: 581-582.

(18) FLORES D, GONZÁLEZ J, ROBLEDO T, RUBIO J, ESPIGA I, SAIZ I. Plan Europeo de Actuación sobre Alcohol 2000-2005 - Organización Mundial de la Salud Europa. Dirección General de Salud Pública y Consumo, Subsecretaría de Sanidad y Consumo, Ministerio de Sanidad y
Consumo. Madrid, 2000.

(19) Ministerio de Sanidad y Consumo. Declaración sobre Jóvenes y Alcohol (Estocolmo, 2001) (citado 7 de sept. 2001). www.msc.es/salud/ epidemiologia/home.htm.

(20) SAN MOLINA L (1996) " ¿Tiene el alcohol algún efecto beneficioso?". Med Clin 107: 655-656.

(21) Diario Oficial de las Comunidades Europeas. Recomendación del Consejo, de 5 de junio de 2001, sobre el consumo de alcohol por parte de los jóvenes y, en particular, de los niños y adolescentes. DOCE núm L 161, 16/6/2001. 